
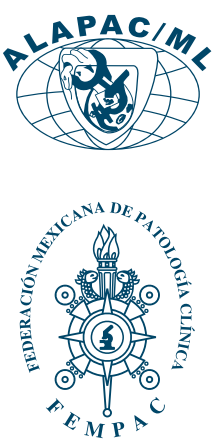

Palabras clave: Pandemia, COVID-19, letalidad.

Keywords:

Pandemic, COVID-19, lethality.

* Director del Programa Integral de Ensayos de Aptitud de la Federación Mexicana de Patología Clínica.

‡ Profesora Emérita, Facultad de Contaduría y Administración, Universidad Nacional Autónoma de México. Miembro del Consejo de Honor de la Federación Mexicana de Universitarias. $\S$ Presidente de la Federación Mexicana de Patología Clínica.

Programa Integral de Ensayos de Aptitud de la Federación Mexicana de Patología Clínica. Ciudad de México.

Correspondencia:

Jesús I Simón

Domínguez

Av. 20 de Noviembre

Núm. 82, interior 205 ,

Colonia Centro, 06090,

Ciudad de México.

Tel: 55-5522-1282

E-mail: jsimon@

piensa.org.mx

Recibido:

07/06/2020

Aceptado:

16/06/2020

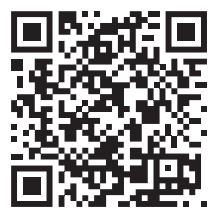

\title{
Cómo estimar la letalidad del COVID-19
}

\author{
How to estimate the lethality of COVID-19
}

\author{
Simón Domínguez Jesús I,* Simón Domínguez Nadima, ${ }^{\ddagger}$ Reyes Núñez Miguel A ${ }^{\S}$
}

RESUMEN

El presente documento tiene como objeto dar a conocer un modelo sencillo y empírico para estimar la letalidad del COVID-19y para responder la pregunta: ¿Cuál es la cantidad de infectados por el SARS-CoV-2 en cada país? Material y métodos: Se realizó una revisión de los estudios previos, de los factores de riesgo que influyen en una mayor mortalidad y el número de defunciones y se concluyó seleccionar un parámetro de letalidad base para las simulaciones con el modelo propuesto, utilizando la base de datos del Centro Europeo para la Prevención y el Control de Enfermedades (ECDC), accesible en la plataforma: https://ourworldindata.org/coronavirus, a partir del 1/4/2020 y con actualizaciones diarias. Resultados: La letalidad seleccionada fue $0.9524 \%$, siendo la media de $1.02 \%$, con un rango de $0.65-1.34 \%$ (149 países). Para validar nuestro modelo se compararon las cifras obtenidas con la simulación contra las reportadas por Rusia (país con más pruebas por habitantes $6.45 \%$ ), con los siguientes resultados para el día 2/5/2020: para casos confirmados, nuestra estimación tuvo una diferencia de $-0.44 \%$; para letalidad de $+0.01 \%$ y para prevalencia de $0.00 \%$. Conclusiones: Nuestro modelo es útil para estimar la letalidad, la prevalencia y sus variaciones de un país a otro, lo cual es esencial para apoyar a los gobiernos en la elección de estrategias apropiadas y evitar incertidumbre en la población.

\section{ABSTRACT}

The purpose of this document is to present a simple and empirical model to estimate the fatality of COVID-19 and to answer the question: ¿What is the number of people infected with SARS-CoV-2 in each country? Material and methods: $A$ review of the previous studies was carried out, of the risk factors that influence a higher mortality, the number of deaths, and it was concluded to select a lethality parameter for the simulations with the proposed model, using the database of the European Center for Disease Prevention and Control, accessible on the platform: https://ourworldindata.org/coronavirus, as of 1/4/2020 and with daily updates. Results: Lethality was adjusted to $0.9524 \%$, the mean being $1.02 \%$, with a range of $0.65-1.34 \%$ (149 countries). To validate our model, the figures obtained with the simulation were compared with those reported by Russia (the country with the most tests per inhabitants 6.45\%), with the following results for the day 2/5/2020: for confirmed cases, our estimate had a difference of $-0.44 \%$; for lethality of $+0.01 \%$ and for prevalence of $0.00 \%$. Conclusions: Our model is useful for estimating case fatality, prevalence and its variations from one country to another, which is essential to support governments in choosing appropriate strategies and avoid population uncertainty.

\section{INTRODUCCIÓN}

T os coronavirus humanos ( $\mathrm{HCoV})$ se han considerado patógenos intrascendentes durante mucho tiempo, son los que causan el «resfriado común»en personas sanas. Sin embargo, en el presente siglo dos $\mathrm{HCoVs}$ altamente patógenos, el coronavirus del síndrome respiratorio agudo severo (SARS-CoV) y el coronavirus del síndrome respiratorio del Medio Oriente (MERS-CoV) demostraron que los reservorios animales pueden causar epidemias globales con morbilidad y mortalidad alarmantes. ${ }^{1}$

El 21 de diciembre de 2019, los médicos chinos trataron a docenas de enfermos de neumonía por causas desconocidas. Se emitió un «aviso urgente sobre el tratamiento de la neumonía de causa desconocida» al Centro Municipal de Salud de Wuhan; en diciembre de 2019 se reconoció en Wuhan, China otro HCoV patógeno, el nuevo coronavirus 2019 (actualmente se denomina SARS-CoV-2), que ha causado grandes brotes, enfermedad grave y la muerte de miles de pacientes. ${ }^{2}$

El 3 de enero de 2020, el gobierno de la República Popular de China informó a la Organización Mundial de la Salud (OMS) sobre la epidemia; el 10 de enero de 2020, investigadores de la Universidad de Fudan, Shanghai publicaron los datos de la secuenciación genética del coronavirus y determinaron que el 
virus es de la misma familia que el coronavirus del SARS. Se compartió con la OMS las secuencias completas del genoma del virus SARS-CoV-2.

El 30 de enero de 2020, el Comité de Emergencias de la OMS llega a un consenso y declara que el brote constituye una emergencia de salud pública de importancia internacional (ESPII); la OMS caracterizó a esta entidad (la denominó COVID-19) como una pandemia el 11 de marzo de $2020 .^{3}$

El 28 de febrero de 2020 se confirma el primer caso en México.

El alcance y efecto final de estos brotes no está claro en la actualidad, ya que la situación está evolucionando rápidamente; hasta el 27 de mayo de 2020 se han documentado 5,656,615 casos confirmados y 355,355 defunciones en 218 países y/o regiones. ${ }^{4}$

Hasta este momento no existen vacunas ni terapias específicas.

$80 \%$ de los casos se resuelven sin recurrir al hospital, $15 \%$ tienen enfermedad grave y requiere de hospitalización, 5\% llegan a un estado crítico que requiere terapia intensiva y apoyo de ventilación mecánica. ${ }^{5}$

Los pacientes mayores de 65 años, del género masculino y las mujeres embarazadas tienen mayor riesgo de presentar casos fatales.

Existen datos documentados que muestran que la comorbilidad (diabetes, obesidad, enfermedades cardiovasculares, hipertensión y otras) es un factor de riesgo de presentar casos graves y críticos; ${ }^{5}$ asimismo, se reporta que los menores de edad tienen menos probabilidades de ser infectados, que el periodo de incubación varía de 2.1 a 11.1 días, con media de 6.4 días $^{5}$ y que existe transmisión en portadores asintomáticos.

Sin embargo, la historia natural de esta enfermedad no se conoce en su totalidad, se necesitan con urgencia estudios en humanos sobre este campo.

La prueba de oro para el diagnóstico de COVID-19 es la reacción en cadena de la polimerasa de transcripción inversa (rRT-PCR) que permite la identificación directa del coronavirus. ${ }^{6}$

La decisión de realizar la prueba molecular debe basarse en factores clínicos, epidemiológicos y vincularse a una evaluación de la probabilidad de infección. Se recomienda realizar esta prueba en todos los casos clínicamente sospechosos de estar infectados por este virus, en los pacientes que cursen con neumonía, graves o críticos, en quienes hayan estado en contacto con un caso confirmado o bajo investigación epidemiológica a COVID-19.

Para el diagnóstico no se recomiendan las pruebas serológicas que cuantifican anticuerpos IgM, IgG e IgA porque representan un riesgo de arrojar falsos negativos, lo cual se reporta generalmente cuando las muestras se toman en el periodo de incubación y en la primera semana de la presencia de síntomas. ${ }^{7}$ No obstante, estas pruebas son de gran utilidad para el seguimiento de los pacientes y estudios epidemiológicos, por lo que habrá un incremento en su uso al término de la pandemia; ${ }^{6}$ dichas pruebas serológicas también son útiles para seleccionar a los pacientes que requieran vacunación (cuando se tenga).

Aun cuando se desconoce el número total de casos de COVID-19, puede afirmarse que el número total de casos de COVID-19 es mayor que el número de casos confirmados conocidos. Esto se debe principalmente a pruebas limitadas, por lo general sólo se realizan en los casos sospechosos, dado que al no tener terapia específica ni vacunas, no ofrece beneficios adicionales.

Los estudios epidemiológicos para determinar la incidencia al término de la epidemia se efectúan con modelos muestrales que cuantifican los anticuerpos específicos contra el SARS-CoV-2 (IgG e IgM).

Durante un brote de enfermedad, lo que merece atención es el crecimiento de la tasa de mortalidad (número de defunciones/número de habitantes), ya que es la única forma actual para hacer comparaciones; por lo anterior, la tasa de letalidad (defunciones/infecciones), que depende del número de habitantes infectados, no se considera útil en este momento de la pandemia porque no se tienen datos reales. Sin embargo, se considera que la letalidad es un estadístico muy importante para evaluar el resultado de la terapia que reciben los casos graves y críticos.

Existe una variedad de medicamentos reutilizados y nuevos para el tratamiento del COVID-19 que están en investigación; asimismo, se realizan cientos de ensayos clínicos con remdesivir, cloroquina, favipiravir, hidroxicloroquina, plasma de pacientes convalecientes y otros que están planificados o en curso, cuya rápida finalización es fundamental para identificar terapias realmente efectivas. Cabe señalar que hasta este momento no existe una terapia validada y autorizada, ya que la evaluación de los agentes requiere tiempo para realizar ensayos controlados, aleatorizados y con criterios de elegibilidad realistas y una estratificación adecuada de los pacientes. ${ }^{8-10}$

Los esfuerzos de investigación previos para desarrollar una vacuna contra el coronavirus del síndrome respiratorio agudo severo (SARS-CoV) en los años posteriores a la pandemia de 2003 han abierto la puerta a los investigadores para diseñar conceptos y enfoques de vacunas para la pandemia de COVID-19, actualmente cerca de 100 organizaciones trabajan para su desarrollo. Es posible que a finales de este año ya estén disponibles. ${ }^{11}$ 


\section{Objetivo}

El objetivo del presente modelo es estimar la letalidad de la pandemia del COVID-19 con base en el número de defunciones y en los factores de riesgo que influyen en la mortalidad.

\section{MATERIAL Y MÉTODOS}

Para conformar la base de datos se utilizó Excel®.

La información requerida se obtuvo de la base de datos de ECDC y de la OMS. ${ }^{3}$

El modelo desarrollado se basó en los resultados de los estudios previos, los factores de riesgo que influyen en la mortalidad y el número de defunciones, según se detalla a continuación:

- Estudios previos: se realizó una revisión de la literatura especializada a partir del 1 de enero de 2020 con el buscador Google académico (palabras clave: letalidad, COVID-19 y pandemia), dado que la información disponible en general son trabajos no evaluados por pares y/o pendientes de publicación debido al poco tiempo seleccionado. Se revisaron un total de 148 artículos, de los cuales se descartaron los artículos que no tenían como objetivo calcular o estimar la letalidad del COVID-19 (total de seleccionados 24). ${ }^{11-35}$

- Factor de riesgo total: se consideraron las prevalencias de diabetes, obesidad, enfermedad cardiovascular y población mayor de 65 años y el factor de riesgo total se calculó con la suma de las diferencias de cada prevalencia por país y con la media de todos los países.

- El número de defunciones se obtuvo de la base de datos de la ECDC.

- La información se actualizó diariamente.

\section{RESULTADOS}

Los resultados de la revisión de la literatura muestran que no existe un consenso en la estimación de la letalidad del COVID-19, ya que los diferentes modelos reportaron un rango de letalidad de 0.2 a $9.8 \%$; con base en lo anterior, se seleccionó un parámetro inicial de la letalidad de $0.9524 \%$ (es decir, que se requieren 105 casos de infectados para una defunción), el cual se ajustó con el factor de riesgo total calculado para cada país.

Se calculó la media de las prevalencias de todos los países seleccionados (149), siendo de $7.71 \%$ para diabetes, $18.81 \%$ para obesidad y $8.7 \%$ para la población mayor de 65 años; para las enfermedades cardiovasculares se utilizó la tasa de mortalidad por 100,000 habitantes, la cual se recalculó en porcentaje con el resultado de $2.3 \%$.

Factor para calcular el número de infectados $(\mathrm{FCNI})=$ (105-(105*factor total de riesgo)).

La letalidad ajustada $=1 / \mathrm{FCNI}$.

Ejemplo para México: número de defunciones el 2 de mayo de 2020: 1,972.

Estimación de casos infectados: 1,972*92.4 =182,213.

Letalidad $=1 / \mathrm{FCNI}=1 / 92.4=1.08 \%$.

Prevalencia $=$ casos infectados/número de habitantes.

Prevalencia $=182,213 / 128,932,753=0.14 \%$.

En la Tabla 1 se presenta FCNI para cada país.

\section{VALIDACIÓN}

- Se realizaron simulaciones y análisis de datos diarios con la base de datos desarrollada, para evaluar concordancias entre los datos registrados y los datos estimados.

- Rusia es el país con más pruebas moleculares realizadas por habitantes $(6.45 \%)$, por lo cual se utilizó para validar nuestro modelo, esperando encontrar concordancias transitorias.

- El 2 de mayo de 2020 Rusia reportó los siguientes datos: número de habitantes $=145,934,460$; número de defunciones $=1,169$; casos confirmados 114,431; letalidad $=1.02 \%$; prevalencia $=0.08 \%$

- Los cálculos realizados con nuestro modelo para Rusia en dicha fecha fueron: factor de riesgo total $=$ 7.19\%. Número de casos infectados: 1,169*(105$(105 * 7.19 \%))=1,169 * 97.5=113,920$.

Cabe destacar que la diferencia de nuestra estimación de casos infectados comparada con los casos confirmados que reportó Rusia fue sólo de 511; Rusia reportó una letalidad de $1.02 \%$ y la calculada con nuestras estimaciones fue de $1.03 \%$, con una diferencia de $0.01 \%$. La diferencia entre la prevalencia estimada y la reportada por Rusia fue muy cercana a $0 \%$, por el redondeo de cifras.

- Se realizó una correlación entre los casos infectados confirmados de Rusia y los casos infectados estimados por nuestro modelo, obteniendo un índice de correlación de: 0.995, (del 27/03/2020 al 6/06/2020).

\section{CONCLUSIONES}

- Los resultados obtenidos son útiles para estimar el número de casos infectados y, por lo tanto, la letalidad y prevalencia de la pandemia del COVID-19. 
Tabla 1: Factor de letalidad ajustada para estimar los casos infectados por país.

\begin{tabular}{|c|c|c|c|c|c|}
\hline País & FCNI & País & FCNI & País & FCNI \\
\hline Afghanistan & 125.0 & Finland & 90.1 & Panama & 98.0 \\
\hline Algeria & 103.4 & France & 89.9 & Peru & 107.5 \\
\hline Argentina & 96.8 & Germany & 87.2 & Philippines & 124.0 \\
\hline Australia & 89.5 & Greece & 89.7 & Poland & 89.8 \\
\hline Austria & 94.4 & Honduras & 112.4 & Portugal & 86.3 \\
\hline Bangladesh & 124.1 & Hungary & 87.0 & Romania & 88.2 \\
\hline Belarus & 93.6 & India & 119.6 & Russia & 97.5 \\
\hline Belgium & 95.3 & Indonesia & 123.3 & Saudi Arabia & 84.4 \\
\hline Bolivia & 111.2 & Iran & 99.9 & Serbia & 90.1 \\
\hline Bosnia and Herzegovina & 93.4 & Iraq & 107.2 & Slovenia & 85.9 \\
\hline Brazil & 103.6 & Ireland & 96.0 & South Africa & 103.8 \\
\hline Bulgaria & 86.3 & Israel & 96.1 & South Korea & 118.3 \\
\hline Cameroon & 121.0 & Italy & 88.4 & Spain & 86.8 \\
\hline Canada & 85.4 & Japan & 104.6 & Sudan & 114.0 \\
\hline Chile & 92.0 & Kuwait & 83.1 & Sweden & 93.3 \\
\hline China & 112.8 & Luxembourg & 96.7 & Switzerland & 95.3 \\
\hline Colombia & 104.9 & Malaysia & 104.1 & Turkey & 90.1 \\
\hline Croatia & 88.4 & Mexico & 92.4 & Ukraine & 93.1 \\
\hline Czech Republic & 84.3 & Morocco & 103.8 & United Arab Emirates & 86.0 \\
\hline Denmark & 93.1 & Netherlands & 94.3 & United Kingdom & 87.3 \\
\hline Dominican Republic & 101.7 & Nigeria & 126.6 & United States & 78.1 \\
\hline Ecuador & 110.2 & Norway & 93.2 & World & 105.1 \\
\hline Egypt & 88.0 & & & & \\
\hline
\end{tabular}

Sólo se incluyen los países con más de 100 defunciones. Casos estimados infectados: número de defunciones por el FCNI del país.

- Las estimaciones realizadas pueden ser diferentes a la realidad, pero los datos reales sólo podrán conocerse hasta tener los resultados de los estudios de serología (presencia de anticuerpos IgM e IgG), los cuales se realizan al término de los brotes.

- El modelo propuesto se validó con las cifras reportadas por Rusia, encontrándose que nuestras estimaciones no presentaron diferencias significativas con aquéllas.

- La letalidad estimada es una constante por país, porque hasta este momento no existe un tratamiento específico y eficaz y los protocolos para tratamiento de los casos graves y críticos son similares; por esto, se considera que el incremento del número de defunciones no necesariamente incrementa la letalidad.

- La prevalencia estimada sí se incrementa con el número de defunciones en forma proporcional.

- Los registros de casos confirmados en este momento no son recomendables para calcular la letalidad, porque producen datos erróneos debido a diferentes criterios para su indicación o por razones económicas, lo cual produce incertidumbre en la población.
- La letalidad ajustada entre todos los países tuvo una media de $1.02 \%$, con un rango de $0.65 \%$ 1.34\% (149 países).

\section{REFERENCIAS}

1. Wu P, Hao X, Lau EHY et al. Real-time tentative assessment of the epidemiological characteristics of novel coronavirus infections in Wuhan, China, as at 22 January 2020. Euro Surveill. 2020; 25 (3): 2000044. Available in: https://www.ncbi.nlm.nih.gov/pmc/articles/ PMC6988272/

2. Chan KW, Wong VT, Tang SCW. COVID-19: an update on the epidemiological, clinical, preventive and therapeutic evidence and guidelines of integrative Chinese-western medicine for the management of 2019 Novel Coronavirus Disease. Am J Chin Med. 2020; 48 (3): 737-762. Available in: https://www.ncbi.nlm.nih.gov/ pubmed/32164424

3. WHO Director-General's opening remarks at the media briefing on COVID-19 [11 March 2020]. Available in: https://www.who. $\mathrm{int} / \mathrm{dg} / \mathrm{speeches/detail/who-director-general-s-opening-remarks-at-}$ the-media-briefing-on-covid-19---11-march-2020

4. Roser M, Ritchie H, Ortiz-Ospina E, Hasell J. Coronavirus Pandemic (COVID-19). Published online at OurWorldInData.org. 2020. Retrieved from: https://ourworldindata.org/coronavirus

5. Rothan HA, Byrareddy SN. The epidemiology and pathogenesis of coronavirus disease (COVID-19) outbreak. J Autoimmun. 
2020; 109: 102433. Available in: https://doi.org/10.1016/j. jaut.2020.102433

6. Wayne Dimech B. Part 1: key facts in COVID-19 test. WESTGARD WEB April 2020.

7. Lippi G, Plebani M. Laboratory abnormalities in patients with COVID-2019 infection [published online ahead of print, 2020 Mar 3]. Clin Chem Lab Med. 2020. Available in: https://doi.org/10.1515/ cclm-2020-0198

8. Gautret P, Lagier JC, Parola P et al. Hydroxychloroquine and azithromycin as a treatment of COVID-19: results of an open-label non-randomized clinical trial [published online ahead of print, 2020 Mar 20]. Int J Antimicrob Agents. 2020; 105949. Available in: https://doi.org/10.1016/j.ijantimicag.2020.105949

9. Devaux CA, Rolain JM, Colson P, Raoult D. New insights on the antiviral effects of chloroquine against coronavirus: what to expect for COVID-19? Int J Antimicrob Agents. 2020; 55 (5): 105938. Available in: https://doi.org/10.1016/j.ijantimicag.2020.105938

10. Ko WC, Rolain JM, Lee NY et al. Arguments in favour of remdesivir for treating SARS-CoV-2 infections. Int J Antimicrob Agents. 2020; 55 (4): 105933. Available in: https://www.ncbi.nlm.nih.gov/pmc/ articles/PMC7135364/

11. Huang C, Wang Y, Li X et al. Clinical features of patients infected with 2019 novel coronavirus in Wuhan, China. Lancet. 2020; 395: 497-506.

12. Chen N, Zhou M, Dong X et al. Epidemiological and clinical characteristics of 99 cases of 2019 novel coronavirus pneumonia in Wuhan, China: a descriptive study. Lancet. 2020; 395: 507-513.

13. Wang D, Hu B, Hu C et al. Clinical characteristics of 138 hospitalized patients with 2019 novel coronavirus-infected pneumonia in Wuhan, China [published online ahead of print, 2020 Feb 7]. JAMA. 2020; 323 (11): 1061-1069. doi: 10.1001/jama.2020.1585.

14. WHO. WHO Director-General's opening remarks at the media briefing on COVID-19. March 3, 2020. Available in: https:// www.who.int/dg/speeches/detail/who-director-general-sopeningremarks-at-the-media-briefing-oncovid-19---3-march-2020

15. Johns Hopkins Center for Systems Science and Engineering. Coronavirus COVID-19 global cases. 2020. Available in: https:// gisanddata.maps.arcgis.com/apps/opsdashboard/index.html\#/ bda7594740fd40299423467b48e9ecf6

16. CNA. Diamond Princess passenger dies, bringing ship's death toll to seven. March 8, 2020. Available in: https://www.channelnewsasia. com/news/asia/coronavirus-covid19-japandiamond-princessdeaths- 12513028

17. Pappas S. How deadly is the new coronavirus? March, 2020. Available in: https://www.livescience.com/iscoronavirus-deadly.html

18. Munster VJ, Koopmans M, van Doremalen N, van Riel D, de Wit E. A novel coronavirus emerging in China-key questions for impact assessment. N Engl J Med. 2020; 382: 692-694.

19. de Wit E, van Doremalen N, Falzarano D, Munster VJ. SARS and MERS: recent insights into emerging coronaviruses. Nat Rev Microbiol. 2016; 14: 523-534.

20. Fauci AS, Lane HC, Redfield RR. Covid-19 - Navigating the uncharted. N Engl J Med. 2020; 382 (13): 1268-1269. doi: 10.1056/NEJMe2002387.
21. Rajgor DD, Lee MH, Archuleta S, Bagdasarian N, Quek SC. The many estimates of the COVID-19 case fatality rate [published online ahead of print, 2020 Mar 27]. Lancet Infect Dis. 2020. doi: 10.1016/S1473-3099(20)30244-9.

22. Miletto Granozio F. On the problem of comparing COVID-19 fatality rates. Available in: https://arxiv.org/ftp/arxiv/ papers/2004/2004.03377.pdf

23. McFall-Johnsen JK, Lauren Frias M. A third of the global population is on coronavirus lockdown here's our constantly updated list of countries and restrictions. Business Insider. Available in: https:// www.businessinsider.com/countries-on-lockdown-coronavirusitaly-2020-3. Accessed April 6, 2020.

24. Baud D, Qi X, Nielsen-Saines K, Musso D, Pomar L, Favre G. Real estimates of mortality following COVID-19 infection [published online ahead of print, 2020 Mar 12]. Lancet Infect Dis. 2020; S1473-3099(20)30195-X. doi: 10.1016/S1473-3099(20)30195-X.

25. Spychalski P, Błażyńska-Spychalska A, Kobiela J. Estimating case fatality rates of COVID-19 [published online ahead of print, 2020 Mar 31]. Lancet Infect Dis. 2020; S1473-3099(20)30246-2. doi: 10.1016/S1473-3099(20)30246-2.

26. Elisabeth M. Coronavirus: covid-19 has killed more people than SARS and MERS combined, despite lower case fatality rate. BMJ. 2020; 368: m641. https://doi.org/10.1136/bmj.m641.

27. Villa M. COVID-19 and Italy's case fatality rate: ¿what's the catch? Available in: https://www.ispionline.it/sites/default/ files/pubblicazioni/ispi analysis italy covid19 lethality villa mar2020.pdf

28. Rinaldi G, Paradisi M. An empirical estimate of the infection fatality rate of COVID-19 from the first Italian outbreak. medRxiv. 2020. Available in: https://doi.org/10.1101/2020.04.18.20070912

29. De Natale G, Ricciardi V, De Luca G et al. The COVID-19 infection in Italy: a statistical study of an abnormally severe disease. J Clin Med. 2020; 9 (5): E1564. doi: 10.3390/jcm9051564.

30. Oke J, Heneghan C. Global COVID-19 case fatality rates. Oxford, UK: Nuffield Department of Primary Care Health Sciences; 2020. Available online: https://www.cebm.net/global-COVID-19-casefatality-rates/

31. Lauer SA, Grantz KH, Bi Q et al. The incubation period of coronavirus disease 2019 (COVID-19) from publicly reported confirmed cases: estimation and application. Ann Intern Med. 2020; 172 (9): 577-582. doi: 10.7326/M20-0504.

32. Tátrai D, Várallyay Z. COVID-19 epidemic outcome predictions based on logistic fitting and estimation of its reliability. arXiv:2003.14160

33. Baumgaertner E. How deadly is the new coronavirus? Scientists race to find the answer. Feb 12, 2020. [accessed March 19, 2020] Available in: https://www.latimes.com/ science/story/2020-02-11/ how-deadly-iscoronavirus-fatality-rate

34. Wighton K, van Elsland S L. Coronavirus fatality rate estimated by Imperial scientists. Feb 11, 2020. [accessed March 19, 2020] Available in: https://www.imperial.ac.uk/news/195217/coronavirusfatality-rateestimated-imperial-scientists

35. WHO. Diabetes country profiles. 2016. Available in: https://www. who.int/diabetes/country-profiles/en/ 